05.3

\title{
Лимитирующие режимы роста III-V нитевидных нанокристаллов
}

\author{
() В.Г. Дубровский ${ }^{1,2}$, А.С. Соколовский ${ }^{3}$, Н. Hijazi $^{3}$ \\ ${ }^{1}$ Санкт-Петербургский государственный университет, Санкт-Петербург, Россия \\ ${ }^{2}$ Физико-технический институт им. А.Ф. Иофффе РАН, Санкт-Петербург, Россия \\ ${ }^{3}$ Университет ИТМО, Санкт-Петербург, Россия \\ E-mail: dubrovskii@mail.ioffe.ru
}

Поступило в Редакцию 19 мая 2020 г.

В окончательной редакции 19 мая 2020 г.

Принято к публикации 28 мая 2020 г.

\begin{abstract}
Проведен теоретический анализ кинетики роста нитевидных нанокристаллов полупроводниковых соединений III-V по механизму пар-жидкость-кристалл при наличии конкуренции трех процессов: скорости осаждения элемента группы V; поступления в каплю атомов группы III с учетом поверхностной диффузии; нуклеации зародышей на границе жидкость-кристалл. Получено обобщенное выражение для скорости вертикального роста нитевидного нанокристалла, которое может быть лимитировано одним из трех

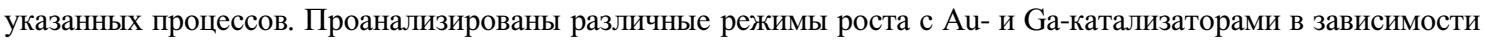
от потоков элементов групп III и V и радиуса нитевидного нанокристалла.
\end{abstract}

Ключевые слова: III-V нитевидный нанокристалл, механизм роста пар-жидкость-кристалл, нуклеация, поверхностная диффузия, соотношение потоков V/III.

DOI: 10.21883/PJTF.2020.17.49889.18384

Полупроводниковые нитевидные нанокристаллы (ННК), в особенности III-V ННК, представляют большой интерес с фундаментальной точки зрения, a также для приложений в наноэлектронике и нанофотонике [1-4]. Большинство III-V ННК выращиваются различными эпитаксиальными методами (молекулярно-пучковая эпитаксия (МПЭ), различные варианты газофазной эпитаксии (ГФЭ)) по механизму пар-жидкость-кристалл (ПЖК) [5], где в качестве катализаторов могут использоваться капли $\mathrm{Au}[5]$ или $\mathrm{Ga}$ при автокаталитическом росте [6]. Одним из несомненных преимуществ ННК над другими типами наноструктур является чрезвычайно эффективная релаксация упругих напряжений на их боковой поверхности $[7,8]$, что позволяет выращивать III-V ННК на рассогласованных подложках кремния, а также гетероструктуры различного типа внутри ННК без формирования дислокаций несоответствия. Для получения ансамблей III-V HНК с заданными свойствами необходимо контролировать скорость их вертикального роста. Этому вопросу было посвящено большое число работ. Известно, что ПЖК-рост III-V ННК может лимитироваться либо скоростью нуклеации на границе жидкость-кристалл под каплей [9], либо скоростью поступления атомов элемента группы III с учетом их поверхностной диффузии с боковой поверхности на вершину ННК $[10,11]$, либо адсорбцией и десорбцией элемента группы V [12]. Автокаталитический рост HНК GaAs из капель $\mathrm{Ga}$ всегда происходит в обогащенных по Ga условиях и является As-лимитированным [4,12]. Ранее комбинированный механизм роста III-V ННК с учетом всех указанных процессов рассматривался в [13-15]. В настоящей работе приводятся вывод простого аналитического выражения для скорости роста III-V ННК по механизму ПЖК и анализ различных лимитирующих режимов роста.

В стационарном режиме Аu-каталитического роста III-V ННК, соответствующем постоянным во времени концентрациям различных элементов в капле, скорость роста $d L / d t$ равна скорости поступления атомов элемента группы V (обозначаемого индексом 5) в каплю за вычетом его десорбции [12,15]:

$$
\frac{d L}{d t}=\frac{4 v_{5(2)}}{1+\cos \beta}\left(1-e^{2 \mu_{5}-\mu_{5(2)}^{v}}\right) .
$$

Здесь $v_{5(2)}$ есть поток димеров группы $\mathrm{V} \quad\left(\mathrm{As}_{2}\right.$ или $\left.\mathrm{P}_{2}\right)[\mathrm{nm} / \mathrm{s}], \beta-$ контактный угол капли, $\mu_{5}-$ химический потенциал атома элемента группы V в капле жидкости, $\mu_{5(2)}^{v}-$ химический потенциал димера в газовой фазе. Общий коэффициент 2 возникает из-за того, что адсорбция димера на поверхности капли дает два атома, член $2 \mu_{5}$ в экспоненте описывает десорбцию в виде димера. Равновесие между жидкостью и паром соответствует условию $2 \mu_{5}=\mu_{5(2)}^{v}$ [12]. Выражение (1) записано для случая ГФЭ. С другой стороны, скорость роста ННК в моноцентрическом режиме нуклеации двумерных зародышей равна [2,9]:

$$
\frac{d L}{d t}=h \pi R^{2} \eta J(\Delta \mu)
$$

Здесь $h$ есть высота монослоя ННК, $R$ - радиус ННК, $J(\Delta \mu)=J_{*}(\Delta \mu) \exp \left(-a^{2} / 4 \Delta \mu\right)$ - скорость нуклеации островков, $a$ - параметр, пропорциональный поверхностной энергии боковой грани островка [16], $\Delta \mu=\mu_{3}+\mu_{5}-\mu_{35}-$ разность химических потенциалов на пару III-V в жидкости и кристалле $\left(\mu_{35}\right)$ [17], 


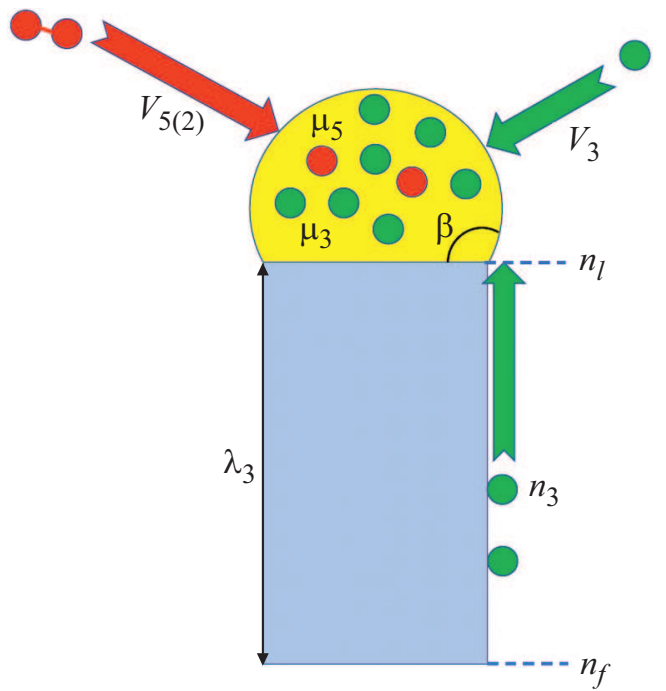

Рис. 1. Иллюстрация модели роста ННК GaAs по механизму ПЖК из потоков $\mathrm{Ga} v_{3}$ и $\mathrm{As}_{2} v_{5(2)}$. Химические потенциалы $\mathrm{Ga}$ и As в капле $\mu_{3}$ и $\mu_{5}$ определяют скорости десорбции (включая обратную диффузию для Ga). Диффузионная длина адатома Ga на боковой поверхности ННК равна $\lambda_{3}$.

$\mu_{3}$ - химический потенциал атома элемента группы III (обозначаемого индексом 3) в капле жидкости; предэкспоненциальный фактор $J_{*}$ слабо зависит от $\Delta \mu$. Величина $\eta$ равна единице в случае, если нуклеация островков происходит на всей верхней грани, и $2 r_{c} / R$, если нуклеация происходит на тройной линии по периметру ННК, где $r_{c}(\Delta \mu)$ есть линейный размер критического зародыша [2].

Рассмотрим теперь выражение для скорости роста ННК через потоки элемента группы III, используя модель, изображенную на рис. 1. Если концентрация адатомов $n_{3}$ на боковой поверхности ННК равна $n_{l}$ на границе с каплей и $n_{f}$ на расстоянии от нее, стандартное выражение для диффузионной составляющей скорости роста ННК имеет вид $[2,11]$ :

$$
\left(\frac{d L}{d t}\right)_{\text {diff }}=\frac{2 \lambda_{3}}{R} v_{3}\left(1-\frac{n_{l}}{n_{f}}\right) .
$$

Отношение $n_{l} / n_{f}$ может быть представлено в виде $n_{l} / n_{f}=\exp \left(\mu_{3}-\mu_{f}\right)$, где $\mu_{f}$ есть химический потенциал адатома на расстоянии $\lambda_{3}$ от капли [18]. В отсутствие латерального роста на боковой поверхности ННК последний должен быть равен химическому потенциалу атомов элемента группы III в газовой фазе: $\mu_{f}=\mu_{3}^{v}$. Отсюда следует

$$
\left(\frac{d L}{d t}\right)_{d i f f}=\frac{2 \lambda_{3}}{R} v_{3}\left(1-e^{\mu_{3}-\mu_{2}^{v}}\right) .
$$

В случае ГФЭ вклад в скорость роста ННК за счет прямого обмена с газом равен

$$
\left(\frac{d L}{d t}\right)_{\text {vapor }}=\frac{2 v_{3}}{1+\cos \beta}\left(1-e^{\mu_{3}-\mu_{3}^{v}}\right) .
$$

Суммируя выражения (4) и (5), получаем

$$
\frac{d L}{d t}=v_{3}\left(\frac{2}{1+\cos \beta}+\frac{2 \lambda_{3}}{R}\right)\left(1-e^{\mu_{3}-\mu_{3}^{v}}\right) .
$$

где равновесие соответствует условию $\mu_{3}=\mu_{3}^{v}$. Таким образом, при известных скоростях осаждения, энергетических и кинетических константах, три уравнения (1), (2) и (6) позволяют определить три неизвестные величины: $\mu_{3}, \mu_{5}$ и $d L / d t$. В случае МПЭ-роста ННК необходим лишь учет геометрии направленных молекулярных пучков, который нетрудно провести на основе результатов работы [19].

Химические потенциалы в капле выражаются через скорость роста ННК согласно выражению

$$
\begin{gathered}
\mu_{3}=\mu_{3}^{v}+\ln \left(1-\frac{1}{V_{3}} \frac{d L}{d t}\right), \\
\mu_{5}=\frac{1}{2} \mu_{5(2)}^{v}+\frac{1}{2} \ln \left(1-\frac{1}{V_{5}} \frac{d L}{d t}\right),
\end{gathered}
$$

где мы используем обозначения

$$
V_{3}=2 v_{3}\left(\frac{1}{1+\cos \beta}+\frac{\lambda_{3}}{R}\right), \quad V_{5}=\frac{4 v_{5(2)}}{1+\cos \beta} .
$$

Используя выражения (7) в формуле (2), получаем замкнутое уравнение на $d L / d t$. Чрезвычайно резкая экспоненциальная зависимость скорости нуклеации от $\Delta \mu$ с большим параметром $a \gg 1$ позволяет использовать асимптотический метод, подробно описанный в [15], и получить решение в виде

$$
\frac{d L}{d t}=\frac{V_{5}}{\left(1+V_{5} / V_{3}\right)\left[1+j_{c} / W(y)\right]}, y=\frac{1+V_{5} / V_{3}}{V_{5}} V_{35} j_{c} .
$$

Скорость роста, лимитированная нуклеацией, определяется выражением

$$
V_{35}=h \pi R^{2} \eta J_{*}\left(\Delta \mu_{v}\right) \exp \left(-a^{2} / 4 \Delta \mu_{v}\right)
$$

и содержит только химический потенциал газовой среды $\Delta \mu_{v}=\mu_{3}^{v}+\mu_{5(2)}^{v} / 2-\mu_{35}$. Величина $j_{c}=\left[a^{2} / 4\left(\Delta \mu_{v}\right)^{2}\right]$ есть число пар III-V в критическом зародыше [2]; $W(y)$ есть функция Ламберта, определяемая согласно выражению $W \exp (W)=y$.

Выражение (9) можно упростить, заметив, что член $j_{c} / W(y)$ в знаменателе лимитирует скорость роста ННК только тогда, когда он много больше единицы. Это имеет место при $W(y) \ll 1$, когда $W(y) \cong y$. Поэтому мы можем использовать приближение

$$
\frac{j_{c}}{W(y)} \cong \frac{j_{c}}{y}=\frac{V_{5}}{\left(1+V_{5} / V_{3}\right) V_{35}} .
$$

Используя это выражение в (9), получаем основной результат работы

$$
\frac{d L}{d t}=\frac{V_{5}}{1+V_{5} / V_{3}+V_{5} / V_{35}} .
$$


Учитывая, что химические потенциалы в газовой среде логарифмически зависят от потоков, имеем $\mu_{3}^{v}=\mu_{3,0}^{v}+\ln \left(v_{3} / v_{3}^{0}\right) \quad$ и $\quad \mu_{5(2)}^{v}=\mu_{5(2), 0}^{v}+\ln \left(v_{5(2)} / v_{5(2)}^{0}\right)$, где индексом 0 помечены потоки, соответствующие значениям $\mu_{3,0}^{v}$ и $\mu_{5(2), 0}^{v}$. Используя это в формуле для $\Delta \mu_{v}$ и считая, что изменение химического потенциала газовой среды мало по сравнению с его абсолютным значением, из (10) имеем

$$
V_{35}=V_{35}^{0}\left[\left(\frac{v_{3}}{v_{3}^{0}}\right)\left(\frac{v_{5(2)}}{v_{5(2)}^{0}}\right)^{1 / 2}\right]^{i_{c}} .
$$

Здесь скорость роста $V_{35}^{0}$ соответствует химическому потенциалу газовой среды $\Delta \mu_{v}^{0}=\mu_{3,0}^{v}+\mu_{5(2), 0}^{v} / 2-\mu_{35}$ и $i_{c}=j_{c}\left(\Delta \mu_{v}^{0}\right)$ есть критический размер при этом значении химического потенциала.

Проанализируем качественно полученное выражение (12) для скорости роста III-V ННК. В условиях, обогащенных по группе III $\left(V_{5} / V_{3} \ll 1\right)$, скорость роста лимитирована потоками элемента группы V: $d L / d t \cong V_{5} /\left(1+V_{5} / V_{35}\right)$ что соответствует, в частности, автокаталитическому росту [6,12]. В противоположном случае $V_{5} / V_{3} \gg 1$ (условия, обогащенные по группе $\mathrm{V}$ ) скорость роста лимитирована потоками элемента группы III: $d L / d t \cong V_{3} /\left(1+V_{3} / V_{35}\right)$, что соответствует, в частности, диффузионному росту $[10,11]$. Данные выражения переходят в скорость роста, лимитированную нуклеацией: $d L / d t \cong V_{35}$ при $V_{5} / V_{35} \gg 1$ или $V_{3} / V_{35} \gg 1$ [9], что имеет место при низких пересыщениях газовой среды (малые $\Delta \mu_{v}$, отвечающие экспоненциальному убыванию скорости нуклеации $J(\Delta \mu))$ или малом радиусе ННК $R$. В противоположном случае $V_{5} / V_{35} \ll 1$ или $V_{3} / V_{35} \ll 1$ (высокие пересыщения газовой среды $\Delta \mu_{v}$, достаточно большой радиус $R$ ) осуществляются режимы роста, лимитированные транспортом элементов групп $\mathrm{V}$ или III в каплю: $d L / d t \cong V_{5}$ или $d L / d t \cong V_{3}$. Таким образом, все известные ранее режимы роста III-V ННК по механизму ПЖК (см. обзор [2]) содержатся в обобщенной формуле (12).

Полученное выражение позволяет рассматривать скорость роста ННК как функцию газовых потоков, температуры и радиуса ННК. Рассмотрим, например, $\mathrm{Au}-$ каталитический рост НHК GaAs при постоянной температуре, фиксированном потоке $\mathrm{As}_{2}\left(V_{5}=V_{5}^{0}\right)$ и различных потоках Ga. Тогда из общих выражений (12), (8) и (13) имеем

$$
\begin{aligned}
\frac{1}{V_{5}^{0}} \frac{d L}{d t}= & \left\{1+\left[F_{53}^{0} /\left(1+\cos \beta \lambda_{3} / R\right)\right]\left(v_{3}^{0} / v_{3}\right)\right. \\
& \left.+\left(R_{0} / R\right)^{2}\left(v_{3}^{0} / v_{3}\right)^{i_{c}}\right\}^{-1} .
\end{aligned}
$$

Характерный „радиус нуклеации“, определенный согласно $R_{0}^{2}=V_{5}^{0} /\left[h \pi J\left(\Delta \mu_{v}^{0}\right)\right]$, экспоненциально убывает при увеличении пересыщения газовой среды. Величина $F_{53}^{0}=2 v_{5(2)}^{0} / v_{3}^{0}$ есть отношение потоков V/III, соответствующее начальному состоянию. В первом приближении мы считаем контактный угол капли $\beta$ не зависящим

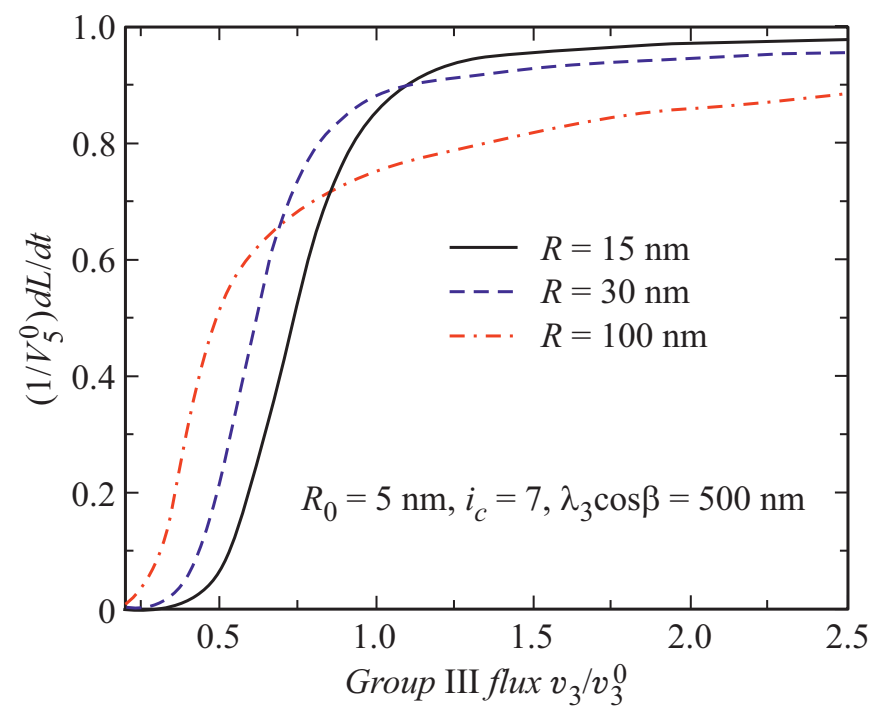

Рис. 2. Зависимости приведенной скорости роста III-V НHК (в единицах потока элемента группы V) с Аu-катализатором от потока элемента группы III при различных радиусах ННК: 15,30 и $100 \mathrm{~nm}$. Расчет проведен по формуле (14) при параметрах, указанных на рисунке, и $F_{53}^{0}=2$.

от соотношения потоков (уточнение можно провести на основе результатов работы [20]).

На рис. 2 представлены зависимости приведенной скорости роста III-V (GaAs) НHК от приведенного потока $v_{3} / v_{3}^{0}$ для трех различных радиусов. При низких потоках $\mathrm{Ga}$ рост лимитируется нуклеацией при любом $R$, при этом широкие ННК растут быстрее узких [9]. При промежуточных потоках Ga происходит переход к $\mathrm{Ga-лимитированному} \mathrm{диффузионному} \mathrm{росту,}$ когда узкие ННК растут быстрее широких [10,11]. При очень высоких потоках $\mathrm{Ga}$ имеет место переход к Asлимитированному росту, при котором скорость роста стремится к скорости осаждения As и перестает зависеть от потока $\mathrm{Ga}$, причем указанный переход происходит быстрее для более узких ННК.

На рис. 3 представлены зависимости приведенной скорости роста III-V (GaAs) ННК от его радиуса при фиксированных потоках обоих элементов и трех различных значениях радиуса нуклеации $R_{0}$. Как и в работах $[14,15]$, при малом $R_{0}=1 \mathrm{~nm}$ во всей интересной области радиусов наблюдается диффузионная (убывающая) зависимость. При промежуточном значении $R_{0}=10 \mathrm{~nm}$ зависимость скорости роста ННК от радиуса становится комбинированной: возрастающий участок кривой при малых радиусах по достижении максимума при $R \sim 30 \mathrm{~nm}$ переходит в убывающий. При большом $R_{0}=100 \mathrm{~nm}$, соответствующем малым значениям пересыщения газовой среды, наблюдается возрастающая зависимость, означающая, что ПЖК-рост лимитирован нуклеацией зародышей [9]. 


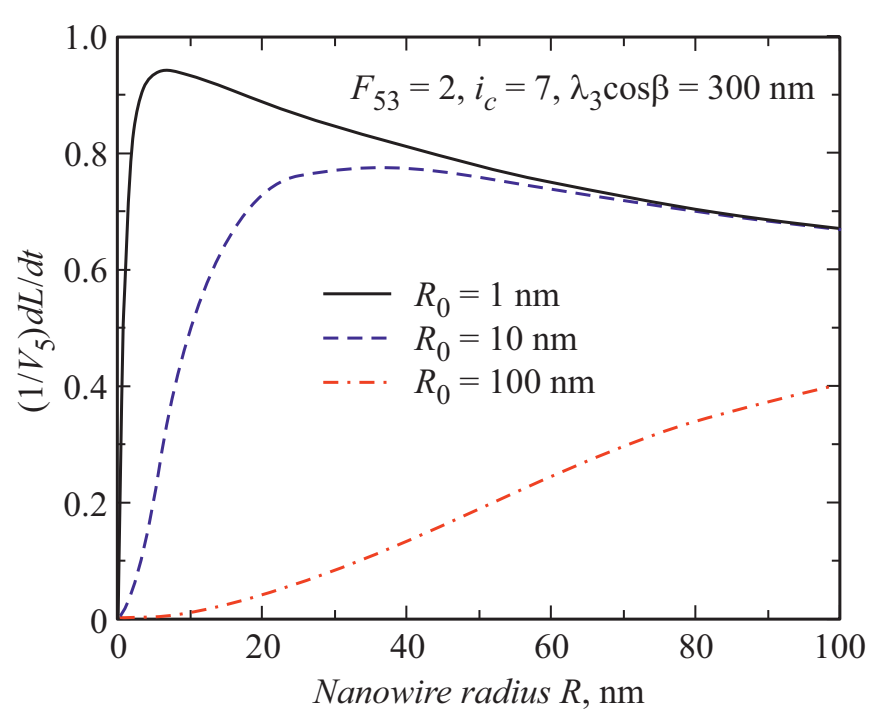

Рис. 3. Зависимости приведенной скорости роста III-V HНК от его радиуса при различных значениях параметра $R_{0}: 1,10$ и $100 \mathrm{~nm}$. Расчет проведен по формуле (14) при параметрах, указанных на рисунке, и $v_{3}=v_{3}^{0}$ (фиксированы потоки обоих элементов).

В заключение отметим, что в работе построена обобщенная модель роста III-V ННК по механизму ПЖК и получено простое выражение для скорости роста ННК, включающее в себя все известные режимы роста: диффузионный, лимитированный потоком элемента группы V и нуклеацией на границе жидкость-кристалл. Полученные результаты позволяют управлять скоростью роста III-V ННК за счет изменения условий эпитаксиального роста (абсолютных значений и соотношения потоков V/III, температуры поверхности и т.д.). Выражения для химических потенциалов атомов элементов групп III и V в капле через скорость роста ННК позволяют избежать их расчетов через удельные концентрации, которые не могут быть измерены для As и Р в силу их высокой летучести и малого содержания в капле (ниже уровня детектирования любыми методами). Это позволяет проводить моделирование различных физикохимических процессов, зависящих от химических потенциалов в жидкой капле, включая процессы легирования III-V ННК.

\section{Финансирование работы}

Работа выполнена при поддержке Российского научного фонда (грант № 19-72-30004).

\section{Конфликт интересов}

Авторы заявляют, что у них нет конфликта интересов.

\section{Список литературы}

[1] Zhang A., Zheng G., Lieber C.M. Nanowires: building blocks for nanoscience and nanotechnology. Springer, 2016. $327 \mathrm{p}$.

[2] Dubrovskii V.G. Theory of VLS growth of compound semiconductors // Semiconductors and semimetals / Eds A. Fontcuberta i Morral, S.A. Dayeh, C. Jagadish. Burlington: Academic Press, 2015. V. 93. P. 1-78.

[3] Dimakis E., Jahn U., Ramsteiner M., Tahraoui A., Grandal J., Kong X., Marquardt O., Trampert A., Riechert $H$., Geelhaar L. // Nano Lett. 2014. V. 14. P. 2604-2609.

[4] Cirlin G.E., Bouravleuv A.D., Soshnikov I.P., Samsonenko Yu.B., Dubrovskii V.G., Arakcheeva E.M., Tanklevskaya E.M., Werner P. // Nanoscale Res. Lett. 2010. V. 5. P. $360-363$.

[5] Wagner R.S., Ellis W.C. // Appl. Phys. Lett. 1964. V. 4. P. 8990.

[6] Matteini F., Dubrovskii V.G., Rüffer D., Tütüncüoğlu G., Fontana Y., Fontcuberta i Morral A. // Nanotechnology. 2015. V. 26. P. 105603 .

[7] Glas F. // Phys. Rev. B. 2006. V. 74. P. 121302(R).

[8] Ng K.W., Ko W.S., Tran T.T.D., Chen R., Nazarenko M.V., Lu F., Dubrovskii V.G., Kamp M., Forchel A., ChangHasnain C.J. // ACS Nano. 2013. V. 7. P. 100-107.

[9] Дубровский В.Г., Сибирев Н.В., Цырлин Г.Э. // Письма в ЖТФ. 2004. Т. 30. В. 16. С. 41-50.

[10] Цырлин Г.Э., Дубровский В.Г., Сибирев Н.В., Сошников И.П., Самсоненко Ю.Б., Тонких А.А., Устинов В.М. // ФТП. 2005. Т. 39. В. 5. С. 587-594.

[11] Dubrovskii V.G., Soshnikov I.P., Sibirev N.V., Cirlin G.E., Ustinov V.M. // J. Cryst. Growth. 2006. V. 289. P. 31-36.

[12] Glas F., Ramdani M.R., Patriarche G., Harmand J.C. // Phys. Rev. B. 2013. V. 88. P. 195304.

[13] Johansson J., Magnusson M.H. // J. Cryst. Growth. 2019. V. 525. P. 125192.

[14] Dubrovskii V.G., Hervieu Yu.Yu. // J. Cryst. Growth. 2014. V. 401. P. 431-440.

[15] Dubrovskii V.G. // J. Cryst. Growth. 2016. V. 440. P. 62-68.

[16] Dubrovskii V.G., Grecenkov J. // Cryst. Growth Design. 2015. V. 15. P. 340-347.

[17] Glas F. // J. Appl. Phys. 2010. V. 108. P. 073506.

[18] Dubrovskii V.G. // Phys. Status Solidi B. 1992. V. 171. P. 345356.

[19] Glas F. // Phys. Status Solidi B. 2010. V. 247. P. 254-258.

[20] Gao Q., Dubrovskii V.G., Caroff P., Wong-Leung J., Li L., Guo Y., Fu L., Tan H.H., Jagadish C. // Nano Lett. 2016. V. 16. P. $4361-4367$. 